\title{
Regression-type Estimators Based on Two Auxiliary Variables of a Finite Population Mean in Two-phase Sampling
}

\author{
P. A. Patel ${ }^{1}$ and F. H. Shah ${ }^{2 *}$ \\ ${ }^{1 *}$ Department of Statistics, Sardar Patel University, Gujarat, India \\ ${ }^{2}$ Department of Statistics, Sardar Patel University, Gujarat, India \\ *Corresponding author: shah_fagun@yahoo.co.in,Tel.: +919712132255 \\ Available online at: www.isroset.org \\ Received:05/Oct/2018, Accepted:14/Oct/2018, Online: 31/Oct/2018
}

\begin{abstract}
This paper suggests a class of regression-type estimators of a finite population mean incorporating auxiliary information on two variables at estimation stage in two-phase sampling. This class of estimators includes many known estimators. Up to the first order of approximation the mean square error (MSE) and optimal MSE are obtained and compared with the MSEs of the estimators included in this paper. Also, an empirical comparison is carried out using a Monte Carlo simulation over three natural populations.
\end{abstract}

Keywords-- Auxiliary variables, mean square error, optimum estimation, regression-type estimator, simulation, two-phase sampling.

\section{INTRODUCTION}

Two-phase sampling is a powerful and cost-effective technique. It was first proposed by Neyman [1] and subsequently discussed by many authors. The basic results on simple ratio and regression estimators of the population mean using single auxiliary variable in two-phase sampling is found in Cochran [2]. Many authors have extended these estimators using the known parameters of $x$. Further extension has been done using two auxiliary variables, see Mohanty [3], Khare and Srivastava [4], Sahoo et al.[5], Singh and Upadhyaya [6], Upadhyaya and Singh [7], Samiuddin and Hanif [8,9], among others.Many often even if $\bar{X}$ is unknown, information on a cheaply ascertainable variable $z$, closely related to $x$ but compared to $x$ remotely related to $y$, is available on all population units. In such situations, various estimators have been proposed, see, e.g., Chand [10], Kiregyera [11,12], Mukerjee et al. [13], Srivastava et al. [14], Sahoo et al. [15], Tracy et al.[16], Singh and Espejo [17], Gupta and Shabbir [18], Singh et al. [19], Shukla et al. [20], Choudhury and Singh [21] among others, where some of them have used the knowledge on mean $\bar{Z}$, standard deviation $\sigma_{z}$, coefficient of variation $C_{z}$, coefficient of skewness $\beta_{1}(z)$, coefficient of kurtosis $\beta_{2}(z)$ or combinations of these parameters. Motivated from this work, in this paper we suggest a class of estimators that includes many known estimators and obtain the lower bound of the approximate variance of this class. We also suggest one optimal estimator and compare it with some available estimators using real data.

Consider a finite population $U=\{1, \ldots, i, \ldots, N\}$ of $N$ identifible units. Let $y$ be the study variable and, $x$ and $z$ be auxiliary variables taking the values $\left(y_{i}, x_{i}, z_{i}\right)$ respectively for the population unit $i$. When the two variables $y$ and $x$ are strongly positively related but no information on $\bar{X}$, the population mean of $x$, is available a two-phase sampling can be used. In this scheme the first-phase sample $s^{\prime}$ of size $n^{\prime}$ is selected from $U$, according to a simple random sampling design and witout 
replacement (srswor), to obtain a good estimator of $\bar{X}$. Given $s^{\prime}$, a second-phase sample $s$ of size $n$ is selected from $s^{\prime}$ acording to srsrwor. Let $\bar{u}^{\prime}=\sum_{s^{\prime}} u_{i} / n^{\prime}, \quad \bar{u}=\sum_{s} u_{i} / n$ and $\bar{v}=\sum_{s} v_{i} / n$ where, $u=(x, y, z), b_{u v}\left(b_{u v}^{\prime}\right)$ denotes the regression coefficient of $u$ and $v$ for subsample (preliminary sample)

Our objective is to estimate the population mean $\bar{Y}=\sum_{U} y_{i} / N$, where $\Sigma_{A}$ deonets $\Sigma_{i \in A}$ for any arbitrary set of units $A \subseteq U$. A more extensive use of available auxiliary information is achived through the regression estimators. The two-phase ordinary regression estimator (Cochan [2]) is given by

$$
\widehat{\bar{Y}}_{\text {Regd }}=\bar{y}+b_{y x}\left(\bar{x}^{\prime}-\bar{x}\right)
$$

where $b_{y x}$ denotes the regression coefficient of $y$ and $x$ for sub-sample $s$. The variance of $\widehat{\bar{Y}}_{\text {Regd }}$ to the first order of approximation is

$$
V\left(\hat{\bar{Y}}_{\text {Regd }}\right) \approx \bar{Y}^{2} C_{y}^{2}\left[f_{2}+f_{3}\left(1-\rho_{y x}^{2}\right)\right]
$$

where, $f_{1}=n^{-1}-N^{-1}, f_{2}=n^{-1}-N^{-1}$, and $f_{3}=f_{1}-f_{2}$

and, $C_{y}$ and $\rho_{y x}$ are the population coefficient of variation of $y$ and regression coefficient of $y$ and $x$ respectively.

Chand [10] suggested a chain ratio estimator

$$
\widehat{\bar{Y}}_{c}=\left(\bar{x}^{\prime} \bar{Z} / \bar{z}^{\prime}\right) \bar{y} / \bar{x}
$$

Kiregyera [11,12] proposed the following three estimators:

$$
\begin{aligned}
& \widehat{\bar{Y}}_{k 1}=\left[\bar{x}^{\prime}+b_{x z}^{\prime}\left(\bar{Z}-\bar{z}^{\prime}\right)\right] \bar{y} / \bar{x} \\
& \widehat{\bar{Y}}_{k 2}=\bar{y}+b_{y x}\left(\bar{x}^{\prime} \bar{Z} / \bar{z}^{\prime}-\bar{x}\right) \\
& \widehat{\bar{Y}}_{k 3}=\bar{y}+b_{y x}\left[\left(\bar{x}^{\prime}-\bar{x}\right)+b_{x z}^{\prime}\left(\bar{Z}-\bar{z}^{\prime}\right)\right]
\end{aligned}
$$

This paper is organized as follows. In next section we propose the class of estimators. Its approximate variance and the optimum variance are obtained in Section III. An empirical comparison is carried out in Section IV. The conclusion is given in Section V.

\section{PROPOSED ESTIMATOR AND THE CLASS OF REGRESSION-TYPE ESTIMATORS}

Here we propose the class of regression-type estimators which is defined as

$$
t_{\text {Regcd }}=\bar{y}+b_{y x}\left[\bar{x}^{\prime} \frac{c \bar{Z}+d}{c \bar{z}^{\prime}+d}-\bar{x}\right]
$$

where $c \neq 0$ and $d$ are either real numbers or the known population summary statistics of the auxiliary variable $z$. The subclass of (7) for specific choice $b_{y x}=\bar{y} / \bar{x}$ is 


$$
t_{R c d}=\bar{y} \frac{\bar{x}^{\prime}}{\bar{x}}\left[\frac{c \bar{Z}+d}{c \bar{z}^{\prime}+d}\right]
$$

These two classes of estimators include the following estimators for various choices of $a$ and $b$ :

$$
t_{R 1}=\frac{\bar{y}}{\bar{x}} \bar{x}^{\prime} \frac{\bar{z}}{\bar{z}^{\prime}}
$$

(Chand [10] )

$$
t_{R 2}=\frac{\bar{y}}{\bar{x}} \bar{x}^{\prime}\left[\frac{\bar{Z}+C_{z}}{\bar{z}^{\prime}+C_{z}}\right]
$$

(Singh and Upadhyaya [6] )

$$
t_{R 3}=\frac{\bar{y}}{\bar{x}} \bar{x}^{\prime}\left[\frac{\beta_{2}(z) \bar{z}+C_{z}}{\beta_{2}(z) \bar{z}^{\prime}+C_{z}}\right]
$$$$
t_{\text {Reg3 }}=\bar{y}+b_{y x}\left[\bar{x}^{\prime} \frac{\beta_{2}(z) \bar{Z}+C_{z}}{\beta_{2}(z) \bar{z}^{\prime}+C_{z}}-\bar{x}\right]
$$

(Upadhyaya and Singh [7] )

$$
t_{R 4}=\frac{\bar{y}}{\bar{x}} \bar{x}^{\prime}\left[\frac{C_{z} \bar{z}+\beta_{2}(z)}{C_{z} \bar{z}^{\prime}+\beta_{2}(z)}\right]
$$

$$
t_{\text {Reg } 4}=\bar{y}+b_{y x}\left[\bar{x}^{\prime} \frac{C_{z} \bar{Z}+\beta_{2}(z)}{C_{z} \bar{z}^{\prime}+\beta_{2}(z)}-\bar{x}\right]
$$

(Upadhyaya and Singh [7] )

$$
t_{R 5}=\frac{\bar{y}}{\bar{x}} \bar{x}^{\prime}\left[\frac{\bar{Z}+\sigma_{z}}{\bar{z}^{\prime}+\sigma_{z}}\right]
$$

$$
t_{\text {Reg5 }}=\bar{y}+b_{y x}\left[\bar{x}^{\prime} \frac{\bar{Z}+\sigma_{z}}{\bar{z}^{\prime}+\sigma_{z}}-\bar{x}\right]
$$

(Singh [22] )

$$
t_{R 6}=\frac{\bar{y}}{\bar{x}} \bar{x}^{\prime}\left[\frac{\beta_{1}(z) \bar{Z}+\sigma_{z}}{\beta_{1}(z) \bar{z}^{\prime}+\sigma_{z}}\right]
$$$$
t_{\text {Reg6 }}=\bar{y}+b_{y x}\left[\bar{x}^{\prime} \frac{\beta_{1}(z) \bar{Z}+\sigma_{z}}{\beta_{1}(z) \bar{z}^{\prime}+\sigma_{z}}-\bar{x}\right]
$$

(Singh [22] )

$$
t_{R 7}=\frac{\bar{y}}{\bar{x}} \bar{x}^{\prime}\left[\frac{\beta_{1}(z) \bar{z}+C_{z}}{\beta_{1}(z) \bar{z}^{\prime}+C_{z}}\right] \quad t_{R e g 7}=\bar{y}+b_{y x}\left[\bar{x}^{\prime} \frac{\beta_{1}(z) \bar{Z}+C_{z}}{\beta_{1}(z) \bar{z}^{\prime}+C_{z}}-\bar{x}\right]
$$

\section{APPROXIMATE VARIANCE}

To obtain the approximate variance of $t_{\text {Regcd }}$, let us write

$$
t_{\text {Regcd }}=g\left(\bar{y}, \mathrm{~b}_{\mathrm{yx}}, \bar{x}^{\prime}, \bar{z}^{\prime}, \bar{x}\right)=g(\underline{\hat{\theta}}) \quad \text { and } \quad \bar{Y}=g\left(\bar{Y}, \beta_{\mathrm{yx}}, \bar{X}, \bar{Z}, \bar{X}\right)=g(\underline{\theta})
$$

where

and

$$
\hat{\theta}_{1}=\bar{y}, \quad \hat{\theta}_{2}=\mathrm{b}_{\mathrm{yx}}, \quad \hat{\theta}_{3}=\bar{z}^{\prime}, \quad \hat{\theta}_{4}=\bar{x}^{\prime}, \quad \hat{\theta}_{5}=\bar{x}
$$

$$
\theta_{1}=\bar{Y}, \quad \theta_{2}=\beta_{\mathrm{yx}}, \quad \theta_{3}=\bar{Z}, \quad \theta_{4}=\bar{X}, \quad \theta_{5}=\bar{X}
$$


Now expanding $g(\underline{\hat{\theta}})$ around $g(\underline{\theta})$ using Taylor linearization technique and using Equation (10.12) given in Stuart and Ord [23], viz

$$
\begin{aligned}
V(g(\underline{\hat{\theta}})) \approx \sum_{i}\left[\frac{\partial g(\underline{\hat{\theta}})}{\partial \hat{\theta}_{i}}\right]_{\underline{\hat{\theta}}=\underline{\theta}}^{2} V\left(\hat{\theta}_{i}\right) \\
\quad+\sum \sum_{i \neq j}\left[\frac{\partial g(\underline{\hat{\theta}})}{\partial \hat{\theta}_{i}} \cdot \frac{\partial g(\underline{\hat{\theta}})}{\partial \hat{\theta}_{j}}\right]_{\underline{\hat{\theta}=\theta}} \operatorname{Cov}\left(\hat{\theta}_{i}, \hat{\theta}_{j}\right)+O\left(n^{-3}\right)
\end{aligned}
$$

we obtain

$$
\begin{gathered}
V\left(t_{\text {Regcd }}\right) \approx V(\bar{y})+\beta_{y x}^{2} V\left(\bar{x}^{\prime}\right)+\beta_{y x}^{2}\left(\frac{\bar{X}}{\bar{Z}}\right)^{2} \theta^{2} V\left(\bar{z}^{\prime}\right)+\beta_{y x}^{2} V(\bar{x})+2 \beta_{y x} \operatorname{Cov}\left(\bar{y}^{\prime}, \bar{x}^{\prime}\right) \\
-2 \beta_{y x} \frac{\bar{X}}{\bar{Z}} \theta \operatorname{Cov}\left(\bar{y}, \bar{z}^{\prime}\right)-2 \beta_{y x} \operatorname{Cov}(\bar{y}, \bar{x})-2 \beta_{y x}^{2} \operatorname{Cov}\left(\bar{x}^{\prime}, \bar{x}\right)
\end{gathered}
$$

where

$$
\theta=c \bar{Z} /(c \bar{Z}+d)
$$

Inserting variances and covariance under two-phase sampling (Singh et al. [24] ) we obtain after simplification

$$
V\left(t_{R e g c d}\right) \approx \bar{Y}^{2} C_{y}^{2}\left[f_{1}\left(1-\rho_{x y}^{2}\right)+f_{2} \rho_{x y}^{2}\right]+f_{2}\left(\Delta^{2} C_{z}^{2}-2 \Delta \bar{Y} \rho_{y z} C_{y} C_{z}\right)
$$

where

$$
\Delta=\beta_{y x} \bar{X} \theta=\beta_{y x} \bar{X}(c \bar{Z}) /(c \bar{Z}+d)
$$

and

$$
f_{1}=n^{-1}-N^{-1}, f_{2}=n^{\prime-1}-N^{-1}, f_{3}=f_{1}-f_{2}
$$

Minimization of (10) gives the optimum value of $\Delta$ as

$$
\Delta_{o p t}=\rho_{y z} \bar{Y} C_{y} / C_{z}
$$

Equivalently (from (11) and (12))

$$
\theta_{o p t}=\rho_{y z} C_{x} / \rho_{y x} C_{z}=\beta_{y z} \bar{Z} / \beta_{y x} \bar{X}=\Lambda \text {, say }
$$

which gives

$$
d_{o p t}=c \bar{Z}(1-\Lambda) / \Lambda \quad \text { or } \quad c_{\text {opt }}=d \Lambda / \bar{Z}(1-\Lambda)
$$

The minimum variance is then given by

$$
\operatorname{minV}\left(t_{\text {Regcd }}\right) \approx \bar{Y}^{2} C_{y}^{2}\left[f_{3}\left(1-\rho_{x y}^{2}\right)+f_{2}\left(1-\rho_{y z}^{2}\right)\right]
$$


Remark 1. Usually $\Lambda$ is unknown and must be estimated using both the samples.

Inserting $\widehat{\Lambda}=b_{y z} \bar{Z} / b_{y x} \bar{x}^{\prime}$ in (13) we obtain the optimal value of $d$ and consequently the optimal estimator as

$$
t_{o p t}=\bar{y}+b_{y x}\left[\bar{x}^{\prime} \frac{\bar{Z}+d_{1}}{\bar{z}^{\prime}+d_{1}}-\bar{x}\right]=\bar{y}+b_{y x}\left[\frac{\bar{x}^{\prime}}{1+\frac{b_{y z}}{b_{y x}}\left(\frac{\bar{z}^{\prime}-\bar{Z}}{\bar{x}^{\prime}}\right)}-\bar{x}\right]
$$

where $d_{1}=\bar{Z}(1-\widehat{\Lambda}) / \widehat{\Lambda}$. (for $\left.c=1\right)$

\section{EMPIRICAL COMPARISON}

This section deals with the empirical comparison of the estimators included in this paper.

\section{A. Empirical comparison under optimality condition}

The minimum variance of the proposed class of estimators given in (14) was compared with the MSEs of the estimators discussed in this paper using three natural populations. A relative efficiency in percentage of each estimator was computed by considering $\hat{\bar{Y}}_{r d}$ as the bench mark estimator.

Data set I: Jobson [25] (The observations are replicated 2 times)

$y$ : Highway Rate

$x$ : Weight

$z$ : Engine size

$N=194, \quad n^{\prime}=80, \quad n=30, \quad \bar{Y}=68.37, \quad \bar{X}=2973.71, \quad \bar{Z}=27.60$,

$\sigma_{z}=12.1286, \quad \rho_{y x}=0.7790, \quad \rho_{y z}=0.7464, \quad \rho_{x z}=0.8862$

$C_{y}=0.1869, C_{x}=0.1761, C_{z}=0.4395, \beta_{1}(z)=0.9441, \beta_{2}(z)=2.5386$

Data set II: Murthy [26]

$y$ : Output for 80 factories in a region

$x$ : Fixed capital

$z$ : Data on number of workers

$N=80, \quad n^{\prime}=40, \quad n=20, \quad \bar{Y}=5182.6, \quad \bar{X}=1126.46, \quad \bar{Z}=285.13$

$\sigma_{z}=270.43, \quad \rho_{y x}=0.9413, \quad \rho_{y z}=0.9149, \quad \rho_{x z}=0.9884$

$C_{y}=0.3542, \quad C_{x}=0.7507, \quad C_{z}=0.9485, \quad \beta_{1}(z)=1.2761, \quad \beta_{2}(z)=3.5808$

Data set III: Fisher [27]

$$
\begin{gathered}
y=\text { Petal width } \\
x=\text { Sepal width } \\
z=\text { Sepal length } \\
N=200, \quad n^{\prime}=80, \quad n=50, \quad \bar{Y}=1.152, \quad \bar{X}=3.208, \quad \bar{Z}=5.78, \\
\sigma_{z}=0.9049, \quad \rho_{y x}=-0.5855, \quad \rho_{y z}=0.8303, \quad \rho_{x z}=-0.4287, \quad C_{y}=0.8229, \\
C_{x}=0.1267, \quad C_{z}=0.1570, \quad \beta_{1}(z)=0.2753, \quad \beta_{2}(z)=2.022
\end{gathered}
$$


Table I. RE (\%) of different estimators with respect to $\bar{Y}_{r d}$

\begin{tabular}{|c|c|c|c|}
\hline Estimator & Data I & Data II & Data III \\
\hline$\hat{\bar{Y}}_{r d}$ & 100.00 & 100.00 & 100.00 \\
\hline$\hat{\bar{Y}}_{\text {regd }}$ & 103.55 & 326.06 & 132.99 \\
\hline$t_{R 1}$ & 52.07 & 63.81 & 114.57 \\
\hline$\hat{\bar{Y}}_{k 1}$ & 133.49 & 87.83 & 95.08 \\
\hline$t_{R e g 1}=\hat{\bar{Y}}_{k 2}$ & 72.77 & 949.62 & 60.44 \\
\hline$\hat{\bar{Y}}_{k 3}$ & 140.31 & 1024.21 & 169.09 \\
\hline$t_{R 2}$ & 53.56 & 64.13 & 114.19 \\
\hline$t_{R 3}$ & 52.66 & 63.90 & 114.38 \\
\hline$t_{R 4}$ & 71.42 & 65.08 & 104.49 \\
\hline$t_{R 5}$ & 90.55 & 118.54 & 112.60 \\
\hline$t_{R 6}$ & 92.48 & 112.71 & 109.27 \\
\hline$t_{R 7}$ & 68.16 & 86.82 & 113.52 \\
\hline$t_{\text {Reg2 }}$ & 74.58 & 952.47 & 61.61 \\
\hline$t_{\text {Reg3 }}$ & 74.58 & 950.43 & 61.02 \\
\hline$t_{R e g 4}$ & 76.86 & 952.62 & 67.37 \\
\hline$t_{\text {Reg } 5}$ & 113.55 & 774.74 & 66.78 \\
\hline$t_{\text {Reg6 }}$ & 115.26 & 844.10 & 79.45 \\
\hline$t_{\operatorname{Reg} 7}$ & 74.69 & 951.86 & 64.54 \\
\hline$t_{\text {Regcd }}$ & 140.59 & 1024.84 & 227.73 \\
\hline
\end{tabular}

\section{B. Comparison using a Monte Carlo simulation}

The estimators $t_{\text {opt }}$ given in (15) and the estimators listed in Sections I and II were compared empirically on three populations given above. For comparison of the estimators, a preliminary sample $s^{\prime}$ of size $n^{\prime}$ was drawn using srswor and a second-phase sample $s$ of size $n$ was drawn using srswor from each of the populations and these estimators were computed. This procedure was repeated $M=5000$ times.For each estimator $t$ its relative percentage bias was calculated as

$$
R B(t)=100 *(\bar{t}-\bar{Y}) / \bar{Y}
$$

and the relative efficiency (in percentage) as

$$
R E(t)=\operatorname{MS} E_{\text {sim }}\left(\hat{\bar{Y}}_{\text {reg }}\right) / M S E_{\text {sim }}(t) X 100
$$

where

$$
\bar{t}=\sum_{j=1}^{M} t_{j} / M \text { and } M S E_{s i m}(t)=\sum_{j=1}^{M}\left(t_{j}-\bar{Y}\right)^{2} /(M-1)
$$


Table II. RB (\%) and RE (\%)

\begin{tabular}{|c|c|c|c|c|c|c|}
\hline \multirow[t]{3}{*}{ Estimator } & \multicolumn{3}{|c|}{ Relative Bias (\%) } & \multicolumn{3}{|c|}{ Efficiency $(\%)$ Relative } \\
\hline & \multicolumn{3}{|c|}{ Population } & \multicolumn{3}{|c|}{ Population } \\
\hline & 1 & 2 & 3 & 1 & 2 & 3 \\
\hline$\hat{\bar{Y}}_{\text {reg }}$ & 0.02 & -2.67 & 0.37 & 100.00 & 100.00 & 100.00 \\
\hline$\hat{\bar{Y}}_{\text {regd }}$ & 0.02 & 0.59 & 0.97 & 100.87 & 272.31 & 143.70 \\
\hline$t_{R 1}=\hat{\bar{Y}}_{C}$ & 0.09 & -1.78 & 0.48 & 51.35 & 63.15 & 109.68 \\
\hline$\hat{\bar{Y}}_{k 1}$ & 0.03 & -2.07 & 0.39 & 133.46 & 90.28 & 96.68 \\
\hline$t_{R e g 1}=\hat{\bar{Y}}_{k 2}$ & 0.04 & 0.89 & 0.14 & 67.59 & 625.29 & 86.39 \\
\hline$\hat{\bar{Y}}_{k 3}$ & 0.02 & 0.78 & 1.08 & 134.06 & 726.46 & 169.14 \\
\hline$t_{R 2}$ & 0.09 & -1.79 & 0.48 & 52.85 & 63.51 & 109.42 \\
\hline$t_{R 3}$ & 0.09 & -1.79 & 0.48 & 51.94 & 63.25 & 109.55 \\
\hline$t_{R 4}$ & 0.06 & -1.81 & 0.41 & 70.80 & 64.61 & 102.99 \\
\hline$t_{R 5}$ & 0.03 & -2.49 & 0.47 & 90.09 & 125.49 & 108.36 \\
\hline$t_{R 6}$ & 0.04 & -2.43 & 0.44 & 92.05 & 119.27 & 106.16 \\
\hline$t_{R 7}$ & 0.08 & -1.79 & 0.47 & 52.94 & 63.44 & 108.81 \\
\hline$t_{R e g 2}$ & 0.04 & 0.89 & 0.17 & 69.29 & 628.06 & 87.53 \\
\hline$t_{R e g 3}$ & 0.04 & 0.89 & 0.16 & 68.26 & 626.07 & 86.96 \\
\hline$t_{R e g 4}$ & 0.02 & 0.88 & 0.73 & 88.46 & 636.08 & 122.65 \\
\hline$t_{\text {Reg } 5}$ & 0.01 & 0.62 & 0.27 & 106.47 & 615.85 & 92.43 \\
\hline$t_{\text {Reg6 }}$ & 0.01 & 0.64 & 0.46 & 108.14 & 660.56 & 103.70 \\
\hline$t_{\operatorname{Reg} 7}$ & 0.04 & 0.88 & 0.22 & 69.39 & 627.46 & 90.33 \\
\hline$t_{o p t}$ & -0.01 & 0.78 & 1.87 & 134.13 & 736.78 & 219.18 \\
\hline
\end{tabular}

Tables I and II prompt the following comments:

(a) The absolute relative bias of each estimator is within reasonable range $\pm 3 \%$.

(b) The suggested class of estimators $t_{R e g c d}$ (with optimum values of $c$ and $d$ ) and in particularly the optimal estimator $t_{\text {opt }}$ given in (15) have performed very well followed by Kiregyera [12] estimator $\hat{\bar{Y}}_{k 3}$.

\section{CONCLUSION}

When partial information on the main auxiliary variable $x$ and complete auxiliary information on the additional auxiliary variable $z$, which is highly positive correlated with $x$, is available and the relation between $y$ and $x$ is a straight line not passing through the origin in this situations our optimal estimator may perform very well. This is reflected in Tables I and II. This is supported by the empirical study presented above. Estimators listed in this paper can be further extended in many ways, e.g., 
using exponential type estimators in ratio method of estimation, using ratio in regression method of estimation, estimation of ratio of two or more study variables, incorporating non-response (Kumar and Kumar [28]) etc.

\section{ACKNOWLEDGMENT:}

We are thankful to the referees and members of the board of editors for their relevant suggestions.

\section{REFERENCES}

[1] J. Neyman, "Contribution to the theory of sampling human populations", J. Amer.Statist.Assoc., 33, pp 101-116, 1938

[2] W. G. Cochran, "Sampling Techniques", John Wiley and Sons, New York, 3rd edition, 1977.

[3] S. Mohanty, "Combination of Regression and Ratio Estimate”, Jour. Ind. Statist. Assoc., 5, pp. 16-19, 1967.

[4] B.B. Khare and S.R.Srivastava, "A general regression ratio estimator for the population mean using two auxiliary variables" Aligarh Journal of Statistics, 1, 1981.

[5] J. Sahoo, L.N. Sahoo and S.Mohanty, "A regression approach to estimation in two phase sampling using two auxiliary variables", Current Sciences, 65, 1, pp 73-75, 1993.

[6] G.N. Singh and L.N. Upadhyay, “A class of modified chain type estimators using two auxiliary variables in two-phase sampling”, Metron, LIII, pp 117-125, 1995.

[7] L.N. Upadhyaya and G.N. Singh, "Chain type estimators using transformed auxiliary variable in two-phase sampling”, Advances in Modeling and Analysis, 38(1-2), pp 1-10, 2001

[8] M. Samiuddin and M. Hanif, "Estimation in two phase sampling with complete and incomplete information", Proc. 8th Islamic Countries Conference on Statistical Sciences, 13, pp 479-495, 2006.

[9] M. Samiuddin and M. Hanif, "Estimation of population mean in single and two phase sampling with or without additional information", Pakistan Journal of Statistics, 23(1), pp 1-9, 2007.

[10] L. Chand, "Some ratio type estimators based on two or more auxiliary variables", Unpublished Ph. D. thesis, Iowa State University, Ames, Iowa (USA), 1975.

[11] B. Kiregyera, “A chain ratio-type estimator in finite population mean in double sampling using two auxiliary variables", Metrika 27, pp 217-223, 1980.

[12] B. Kiregyera, "Regression-type estimator using two auxiliary variables and model of double sampling from finite populations", Metrika 31, pp 215-223, 1984.

[13] R. Mukerjee, T.J. Rao and K. Vijayan, “Regression-type estimators using multiple auxiliary information”, Australian Journal of Statistics, 29 (3): 244254, 1987.

[14] S.R. Srivastava, B.B. Khare, S.R. Srivastava, “A generalized chain ratio estimator for mean of finite population”, Journal of Indian Society of Agricultural Statistics, 42(1):108-117, 1990.

[15] J. Sahoo, L.N. Sahoo and S. Mohanty, "An alternative approach to estimation in two-phase sampling using two auxiliary variables", Biometrical J., 3, pp 293-298, 1994.

[16] D.S.Tracy, H.P.Singh and R. Singh, “An alternative to the ratio-cum-product estimator in sample surveys”, Journal of Statistical Planing and Inference, 53 pp 375-387

[17] H.P. Singh and M.R. Espejo, "Double sampling ratio-product estimator of a finite population mean in sampling surveys", Journal of Applied Statistics 34(1), pp.71-85, 2007.

[18] S. Gupta and J. Shabbir, "On the use of transformed auxiliary variables in estimating population mean by using two auxiliary variables", Journal of Statistical Planning and Inference, 137, pp.1606 - 1611, 2007.

[19] R. Singh, P. Chauhan, N. Sawan, and F. Smarandache, "Improvement in estimating population mean using two auxiliary variables in twophase sampling”, Italian Journal of Pure and Applied Mathematics, N-28, 135-142, 2011.

[20] D. Shukla, S. Pathak and N.S. Thakur, "Estimation of population mean using two auxiliary sources in sample surveys", Statistics in Transition 13(1), pp.21-36, 2012.

[21] S. Choudhury and B.K. Singh, B, "A class of chain ratio-product type estimators with two auxiliary variables under double sampling scheme”, Journal of the Korean Statistical Society 41, pp.247-256, 2012.

[22] G.N. Singh, "On the use of transformed auxiliary variable in estimation of population mean in two-phase sampling”, Statistics in Transition, 5(3), pp 405-416, 2001.

[23] A. Stuart and K. Ord, "Kendall's Advanced Theory of Statistics”, Wiley Publications, Volume 1, Distribution Theory, 4th Edition, 1987.

[24] H.P. Singh, R. Tailor and M.S. Kakaran, “An estimator of Population mean using power transformation”, J.I.S.A.S., 58(2), $223-230,2004$.

[25] J. D. Jobson, “Applied Multivariate Data Analysis”, Springer-Verlag, New York, Vol. II, 1992.

[26] M. N. Murthy, "Sampling theory and methods", Calcutta Statistical Publishing Society, First edition, 1967. 
[27] R.A. Fisher, "The use of multiple measurements in taxonomic problems", Annals of Eugenics, 7:179-188, 1936.

[28] K. Kumar, M. Kumar, "Two Phase Sampling Exponential Type Estimators for Ratio and Product of two Population Means in the Presence of Non-response", International Journal of Scientific Research in Mathematical and Statistical Sciences, Vol.4, Issue.6, pp.26-34, 2017.

\section{AUTHORS PROFILE}

Mr. P. A. Patel completed his M.Sc., M. Phil., Ph. D., from Sardar Patel University, India. He is Professor of Statistics, Department of Statistics, Sardar Patel University, Vallabh Vidyanagar, Gujarat. He specializes in Theory of Sample Surveys and Survey Sampling Inference. He has guided four Ph. D. students and published more than thirty research articles. He has more than thirty three years of teaching experience. Ms. Fagun Shah is currently pursuing her Ph. D. under Mr. P. A. Patel at Sardar Patel University. Her research area is Survey Sampling and shall submit her thesis soon. She has a teaching experience of more than two years with Ahmedabad University. 